\title{
Effect of $\alpha / \beta$ Forging on Microstructure and Texture Inhomogeneity in a Ti-1023 Forged Disk
}

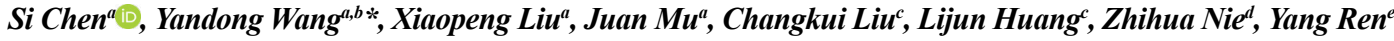 \\ ${ }^{a}$ School of Materials Science and Engineering, Northeastern University, Shenyang 110819, China \\ ${ }^{b}$ School of Materials Science and Engineering, University of Science and Technology Beijing, Beijing \\ 100083, China \\ ${ }^{c}$ Beijing Institute of Aeronautical Materials, Beijing 100095, China \\ ${ }^{d}$ School of Materials Science and Engineering, Beijing Institute of Technology, Beijing 100081, China \\ ${ }^{e}$ X-ray Science Division, Argonne National Laboratory, Argonne, IL 60439, USA
}

Received: November 29, 2018; Revised: June 15, 2019; Accepted: June 25, 2019

\begin{abstract}
The microstructure and texture distribution of a Ti-1023 forged disk were investigated by scanning electron microscopy and synchrotron-based high-energy X-ray diffraction. The finite element method was used to simulate temperature and strain distribution in order to investigate the relationship of the $\alpha / \beta$ forging process with microstructure and texture distribution. A bimodal microstructure and rolling textures with large inhomogeneity were observed in the disk. A plate-like and a necklace-like morphology and volume fraction variations of the primary $\alpha$ phase were observed in different regions with different forging conditions, such as temperature, deformation, position, and high density of flow lines. Texture sharpness distribution of the $\beta$ phase was in good agreement with the strain distribution, which suggests deformation may play the most important part in the texture inhomogeneity. A weak cube texture was obtained near the center of the disk, and fiber textures were found near the rim of the disk. The primary $\alpha$ phase also exhibited a transverse texture, which is favored by large deformation.
\end{abstract}

Keywords: microstructure, texture, forging, synchrotron-based high-energy X-ray diffraction, finite element method.

\section{Introduction}

Near- $\beta$ titanium alloys are suitable for aerospace applications due to their high strength-to-weight ratio and excellent corrosion resistance ${ }^{1,2}$. However, it is still a great challenge to manufacture near- $\beta$ titanium components for aerospace applications, which usually have complex shapes. Different approaches have been made for the manufacture of complex shaped titanium components, such as forging ${ }^{3}$, investment casting ${ }^{4}$, powder metallurgy ${ }^{5}$, and laser rapid prototyping $^{6}$. Although forging is the principal shaping process for the manufacture of titanium components, it is inevitable that inhomogeneous deformation occurs during the forging process due to interface friction ${ }^{7}$, which results in an inhomogeneous microstructure and mechanical properties.

The relationship between forging and the resulting microstructures has been studied extensively in near- $\beta$ titanium alloys $^{8}$. Usually, $\beta$ forging is designed for ingot breakdown and formation of coarse $\alpha$ plates, while the subsequent $\alpha / \beta$ forging aims to achieve the final shape of components with desired microstructure. During this $\alpha / \beta$ forging process, coarse $\alpha$ plates are broken up into individual equiaxed particles ${ }^{9}$. Thus, this process is also called a globularization process. The microstructure evolution of near- $\beta$ titanium alloys is very sensitive to the $\alpha / \beta$ forging processing parameters ${ }^{10}$. Normally, low temperature and large deformation both favor globularization of the $\alpha$ phase. However, texture evolution during the $\alpha / \beta$ forging process is less frequently reported.
Raghunathan et al. ${ }^{11}$ discovered that the initial cube texture was converted into a fiber texture with the increase of strain during $\alpha / \beta$ forging. Gay et al. ${ }^{12}$ observed the presence of large regions of sharp microtexture that varied in different regions of a forged disk.

The aim of this paper is to investigate the microstructure and texture inhomogeneity in a forged disk of the near- $\beta$ titanium alloy Ti-1023 (Ti-10V-2Fe-3Al, $\beta$ transus is $\sim 800^{\circ} \mathrm{C}$ ). Different regions of interest were investigated, and the corresponding microstructures and textures were characterized by scanning electron microscopy (SEM) and synchrotron-based high-energy X-ray diffraction (HE-XRD), respectively. A finite element method (FEM) was used to simulate temperature and strain distribution of the $\alpha / \beta$ forging process to further analyze the effect of the $\alpha / \beta$ forging process on local microstructure and texture variations.

\section{Materials and Methods}

A longitudinal section of a thick forged Ti-1023 disk was analyzed in the current study, which was obtained from Beijing Institute of Aeronautical Materials. The disk was first $\beta$ forged, then closed-die $\alpha / \beta$ forged, and finally solution treated and aged. Flow lines were revealed by macroetching the disk, as shown in Figure 1 (a). Five regions of interest were selected to observe microstructure and texture distribution. Regions 1 and 2 are in the bottom part and in the central part of the disk, respectively, while regions 3 and 5 are close to the rim of the disk. 
Region 4 is located on the minimum cross-section area with a high density of flow lines. Samples of size of $9 \times 4 \times$ $1 \mathrm{~mm}^{3}$ cut from these regions were named S1, S2, etc. The longitudinal direction (LD) and the transverse direction (TD) of the samples are parallel and perpendicular to the flow line in the corresponding region, respectively, as shown in Figure 1 (a).

Optical microscopy (OM) and SEM were applied for microstructure examination, and the HE-XRD technique was used for texture analysis. The HE-XRD technique provides photon fluxes at high energies, making it possible to carry out texture measurements on bulk samples in transmission geometry ${ }^{13}$. Experiments were performed on the 11-ID-C beamline of the Advanced Photon Source at the Argonne National Laboratory. A monochromatic beam with a wavelength of $0.11798 \AA$ and a beam size of $0.2 \times 0.2 \mathrm{~mm}^{2}$ was used. The geometry of HE-XRD texture experiments is illustrated in Figure 1 (b). Samples were mounted on a rotary stage between the incident beam and a two-dimensional (2D) detector (Perkin Elmer amorphous silicon). Diffraction patterns were collected when a sample was rotating around the LD from $-90^{\circ}$ to $90^{\circ}$ with a step size of $15^{\circ}$. A typical diffraction pattern is shown in Figure 1 (b). The intensity variations along the Debye-Scherrer rings indicate the existence of strong texture in the Ti-1023 forged disk. Material Analysis Using Diffraction (MAUD) software ${ }^{14}$ was used to perform the Rietveld refinements for texture analysis.

\section{Results}

\subsection{Microstructure}

Microstructures of five samples characterized by SEM are shown in Figure 2. Generally, every sample presents a bimodal microstructure composed of a primary $\alpha$ phase and a secondary $\alpha$ phase (dark) homogeneously distributed in a $\beta$ matrix (gray). The primary $\alpha$ phase exhibits an equiaxed morphology with an average diameter of $\sim 2 \mu \mathrm{m}$, while the secondary $\alpha$ phase shows a lamellae morphology with a length of $\sim 1 \mu \mathrm{m}$, as shown in Figure 2 (f); the former was formed during the forging process, while the latter was a direct product of post-forging heat treatment. Therefore, it is necessary to assess the differences in the morphology, grain size and volume fraction of the primary $\alpha$ phase among samples to further analyze the effects of the $\alpha / \beta$ forging process on microstructure evolution.

There is an obvious difference in the morphology of the primary $\alpha$ phase between sample 2 and the other samples. Most of the primary $\alpha$ phase with a high aspect ratio shows plate-like rather than equiaxed morphology in S2. As $\alpha$ plates inherited from the $\beta$ forging process were broken up and changed into equiaxed particles during the following $\alpha / \beta$ forging; these remaining $\alpha$ plates indicate a partial recrystallization in S2. Another different morphology observed in S4 is a necklace-like structure with equiaxed $\alpha$ particles distributed along the initial grain boundaries of large $\beta$ grains.

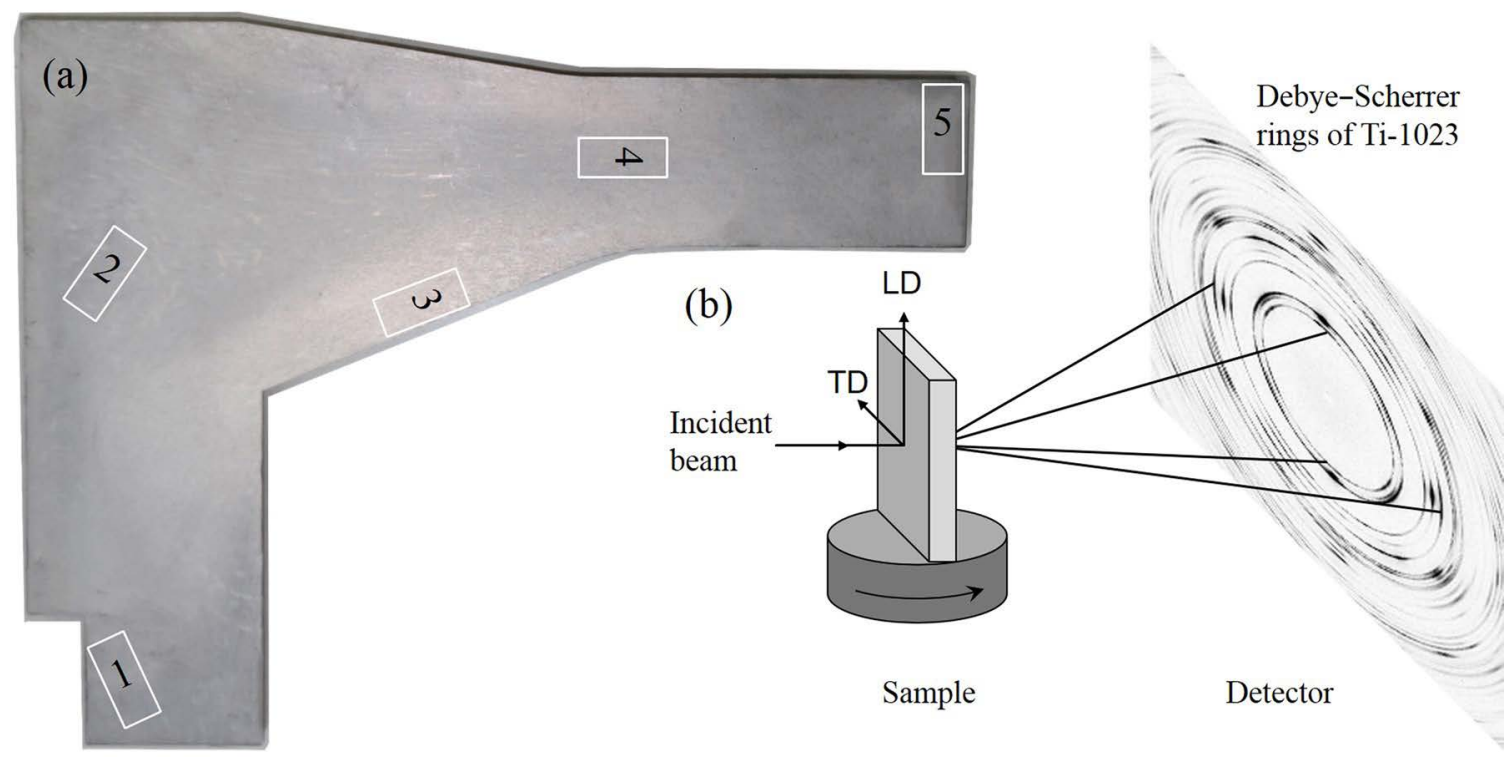

Figure 1. (a) Samples cut from different regions of the Ti-1023 forged disk and the LD of each sample is parallel to the flow line. (b) Schematic geometry of the texture experiments 




Figure 2. SEM micrographs of different samples cut from different regions: (a) S1; (b) S2; (c) S3; (d) S4; (e) S5; (f) typical secondary $\alpha$ phase

For a better evaluation of the grain size and volume fraction of the primary $\alpha$ phase, quantitative metallography was performed on a series of images of different samples obtained by OM. The results are shown in Table 1 . It can be clearly seen that the values of the grain size and volume fraction of S2, i.e., $3.5 \mu \mathrm{m}$ and $27.6 \%$, respectively, are the largest among all samples. Apart from S2, there is no evident difference in the grain size with a small range of $2.28-2.47 \mu \mathrm{m}$ among other samples.
Contrary to this, there is a clear difference in the volume fraction among other samples. The volume fraction of the primary $\alpha$ phase decreases gradually in the other serial samples and reaches the smallest value of $17.3 \%$ in S5.

Table 1. Results of quantitative metallography analysis.

\begin{tabular}{cccccc}
\hline Primary $\alpha$ phase & S1 & S2 & S3 & S4 & S5 \\
\hline Average grain size $(\mu \mathrm{m})$ & 2.28 & 3.5 & 2.33 & 2.41 & 2.47 \\
Volume fraction $(\%)$ & 26.2 & 27.6 & 22.6 & 21.8 & 17.3 \\
\hline
\end{tabular}




\subsection{Texture}

Pole figures of both phases in different samples are shown in Figure 3. The north pole of each pole figure is the LD of each sample, and the right pole is the TD. Shaded contour maps are shown of the reconstructed $\{0002\}_{\alpha},\{10 \overline{1} 0\}_{\alpha}$, $\{10 \overline{1} 1\}_{\alpha},\{100\}_{\beta},\{110\}_{\beta}$, and $\{111\}_{\beta}$ poles. The contours range from 0 to 8 multiples of random with an interval of 1 . When the value of multiples of random equals 1 , the texture is random. Generally, the texture of the $\beta$ phase observed is more distinct than that of the $\alpha$ phase, which indicates texture variations in different samples are dominated by the deformation behavior of the $\beta$ phase.
The pole figures of the $\beta$ phase in S3, S4 and S5 show there is a dominant texture component, $\{100\}<011>$, which is a common rolling texture in body-centered cubic (bcc) materials. Another typical rolling texture component $\{111\}$ $<110>$ also appears in S4, while other texture types in S3 and S5 are difficult to determine just from the distributions of the poles. It is also difficult to determinate the texture type for other two samples. Therefore, more precise characterization for texture type is needed for further discussion. Specially, the texture of the $\beta$ phase in $\mathrm{S} 2$ is much weaker than other samples.

As the primary $\alpha$ phase and the secondary $\alpha$ phase have different thermomechanical history, contribution to the final texture from different types of the $\alpha$ phase should be separated.

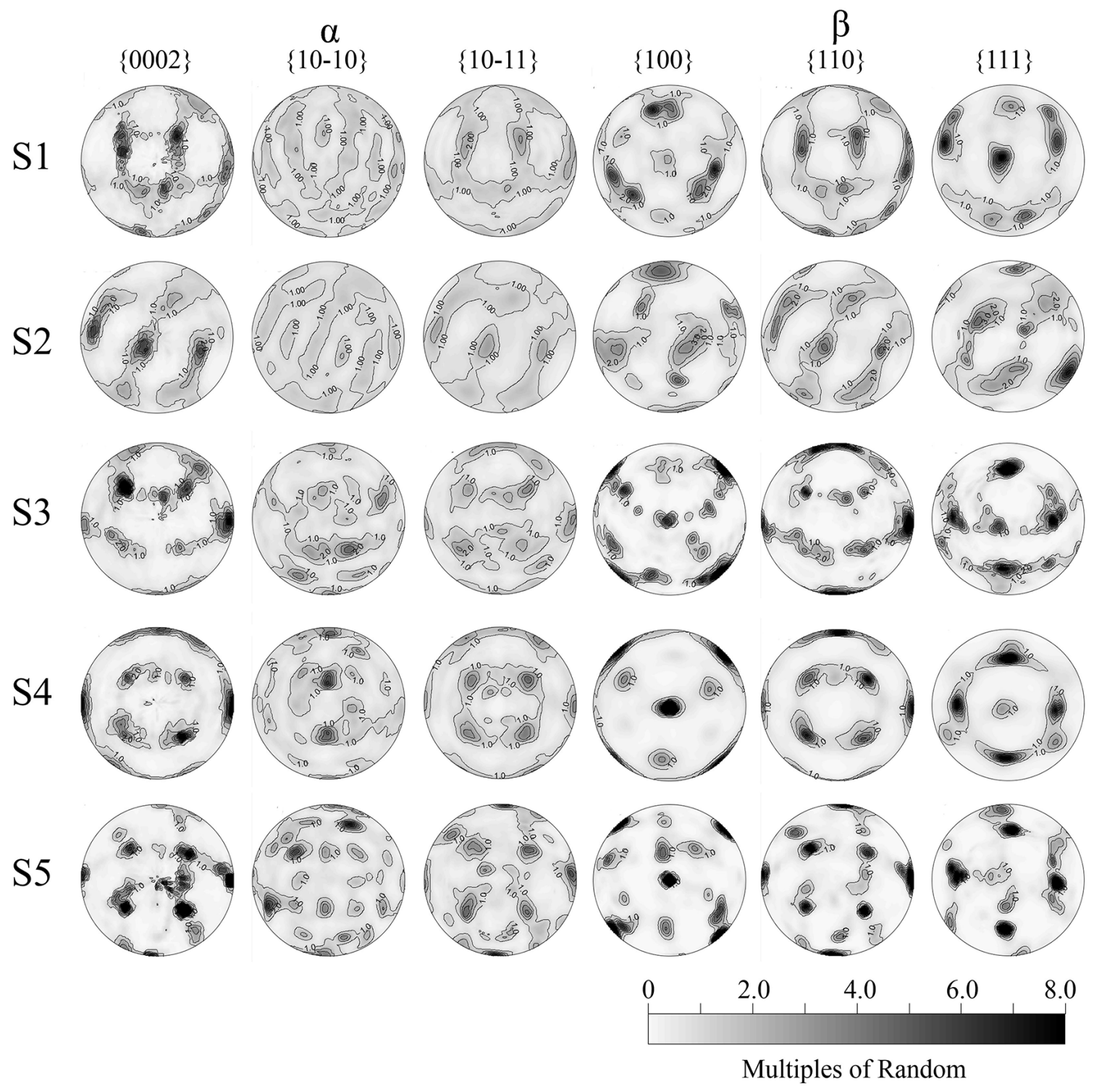

Figure 3. Plot of pole figures from different samples. Equal area projection, upper hemisphere, and pole densities in multiples of random distribution. The north pole of each pole figure is the LD of each sample, and the right pole is the TD 
Using the Rietveld method for texture analysis can also provide other information such as the volume fraction of each phase. The volume fraction of the $\alpha$ phase in different samples are shown in Table 2. By comparing results from Table 1 , it can be easily deduced that the secondary $\alpha$ phase presents an $\sim 50 \%$ portion in all samples. Such a large portion means the secondary $\alpha$ phase makes a significant contribution to the final texture. It is widely known that the secondary $\alpha$ phase is a result of nucleation and growth on the crystallographic planes of the prior- $\beta$ matrix during aging. Thus, according to the Burgers orientation relationship ${ }^{15}$, $\{0002\}_{\alpha}$ planes of the secondary $\alpha$ phase should be parallel to the $\{110\}_{\beta}$ planes resulting in a texture resembling that of $\{110\}_{\beta}$. Thus, the texture of the primary $\alpha$ phase can be separated from the secondary $\alpha$ phase and judged from the $\{10 \overline{1} 0\}_{\alpha}$ and $\{10 \overline{1} 1\}_{\alpha}$ poles. Texture of the primary $\alpha$ phase in S1 and S2 are more random than other samples. In S4, four poles along the RD in the $\{10 \overline{1} 0\}_{\alpha}$ pole and typical $\{10 \overline{1} 1\}_{\alpha}$ pole give the evidence of transverse texture $\{11 \overline{2} 0\}<0001>$. It can also be found in S3 and S5. The transverse texture often forms at high deformation temperature in the $\alpha+\beta$ phase field ${ }^{8}$. This texture will be further discussed in the later sections.

Table 2. The volume fraction and the texture index of each phase in different samples.

\begin{tabular}{ccccc}
\hline \multirow{2}{*}{ Sample name } & \multicolumn{2}{c}{ Volume fraction $(\%)$} & \multicolumn{2}{c}{ Texture index $\mathrm{F}_{2}$} \\
\cline { 2 - 5 } & $\alpha$ & $\beta$ & $\alpha$ & $\beta$ \\
\hline S1 & 67.0 & 33.0 & 5.8 & 10.5 \\
S2 & 75.8 & 24.2 & 2.8 & 7.56 \\
S3 & 79.5 & 20.5 & 8.7 & 153 \\
S4 & 70.0 & 30.0 & 11.7 & 34 \\
S5 & 73.4 & 26.6 & 20.4 & 131 \\
\hline
\end{tabular}

The texture index ${ }^{16}, \mathrm{~F}_{2}$, represents the texture sharpness by a single number, as shown in Table 2. It is equal to the volumeaveraged integral squared orientation distribution function (ODF) values and varies between 1 (random powder) and infinity (perfect texture or single crystal). The results indicate that $\mathrm{F}_{2}$ varies in the range over 2.8-20.4 for the $\alpha$ phase and 7.56-153 for the $\beta$ phase in the disk. Such small values of the $\alpha$ phase indicates a much weaker texture in comparison to the $\beta$ phase. For the $\beta$ phase, the values of $F_{2}$ in S3 and S5, 153 and 131, are an order of magnitude larger than those in other samples.

\subsection{Simulation on $\alpha / \beta$ forging}

As the final microstructure is a strong function of the final $\alpha / \beta$ forging, it is necessary to simulate the $\alpha / \beta$ forging to investigate the origin of the microstructure and texture inhomogeneity. Using the 3D coupled thermalmechanical rigid-plastic finite element method with a slow deformation model, temperature and equivalent plastic strain distribution across the whole disk during the $\alpha / \beta$ forging process has been simulated and is shown in Figure 4.
The locations of different samples in the disk are also marked with numbers. S2 is located near the central part which bears the highest temperature. The second highest temperature by simulation was acquired in the location of S3 and the lowest temperature is in S5. The equivalent plastic strain represents the deformation extent. The value of equivalent plastic strain is approximately 0.56 in S1 and S2, while others are higher. Specially, the strain can reach a rather high value of approximately 1.84 in S3 and S5, which means there is quite a large deformation occurring in these two samples.

It should be noted that the range of temperature variations within the disk is only three degrees and temperature maximum is only $754{ }^{\circ} \mathrm{C}$. This is because the heat coming from the deformation can easily be delivered from the central part to nearby when a slow deformation model is applied to the $\alpha / \beta$ forging simulation. If the strain rate is changed to a much larger value, such as $0.01 / \mathrm{s}$ as reported in Lin's work ${ }^{17}$, the temperature maximum can reach $795^{\circ} \mathrm{C}$, which is quite close to the $\beta$ transus. In practice, this high temperature maximum is made possible because of the poor thermal conductivity and relatively high flow stresses in the $\alpha+\beta$ phase field of titanium alloys.

\section{Discussion}

\subsection{The origin of microstructure inhomogeneity}

Microstructure inhomogeneity observed in the disk mainly embodies the morphology and volume fraction of the primary $\alpha$ phase. Two kinds of different morphology have been observed in this study, and the volume fractions show strong inhomogeneity. The origin of microstructure inhomogeneity is discussed as follows.

The plate-like primary $\alpha$ phase was observed in the S2 located in the region near the center of the disk. From the FEM simulation, this region had been subjected to a high temperature and had undergone a small deformation. It is well known that the aspect ratio of the primary $\alpha$ phase decreases as the deformation strain increases in the globularization process $^{2}$. The high aspect ratio of the primary $\alpha$ phase can be attributed to low deformation in S2. However, S1 also underwent a small deformation but the primary $\alpha$ phase of $S 1$ has a low aspect ratio. Thus, the high temperature of S1 may play a more important role in the morphology. As mentioned before, although the simulated temperature in this region is only $754^{\circ} \mathrm{C}$, a much higher temperature, such as $795^{\circ} \mathrm{C}$, is possible in practice. This high temperature leads to partial recrystallization of the primary $\alpha$ phase and a large amount of the inherited microstructure, i.e., the plate-like morphology.

The necklace-like primary $\alpha$ phase with equiaxed particles along the prior- $\beta$ grain boundaries was found in S4 located in the region with a high density of flow lines. The nucleation sites for globularization can occur in the prior- $\beta$ grain boundaries as well as the prior- $\alpha$ plates. 


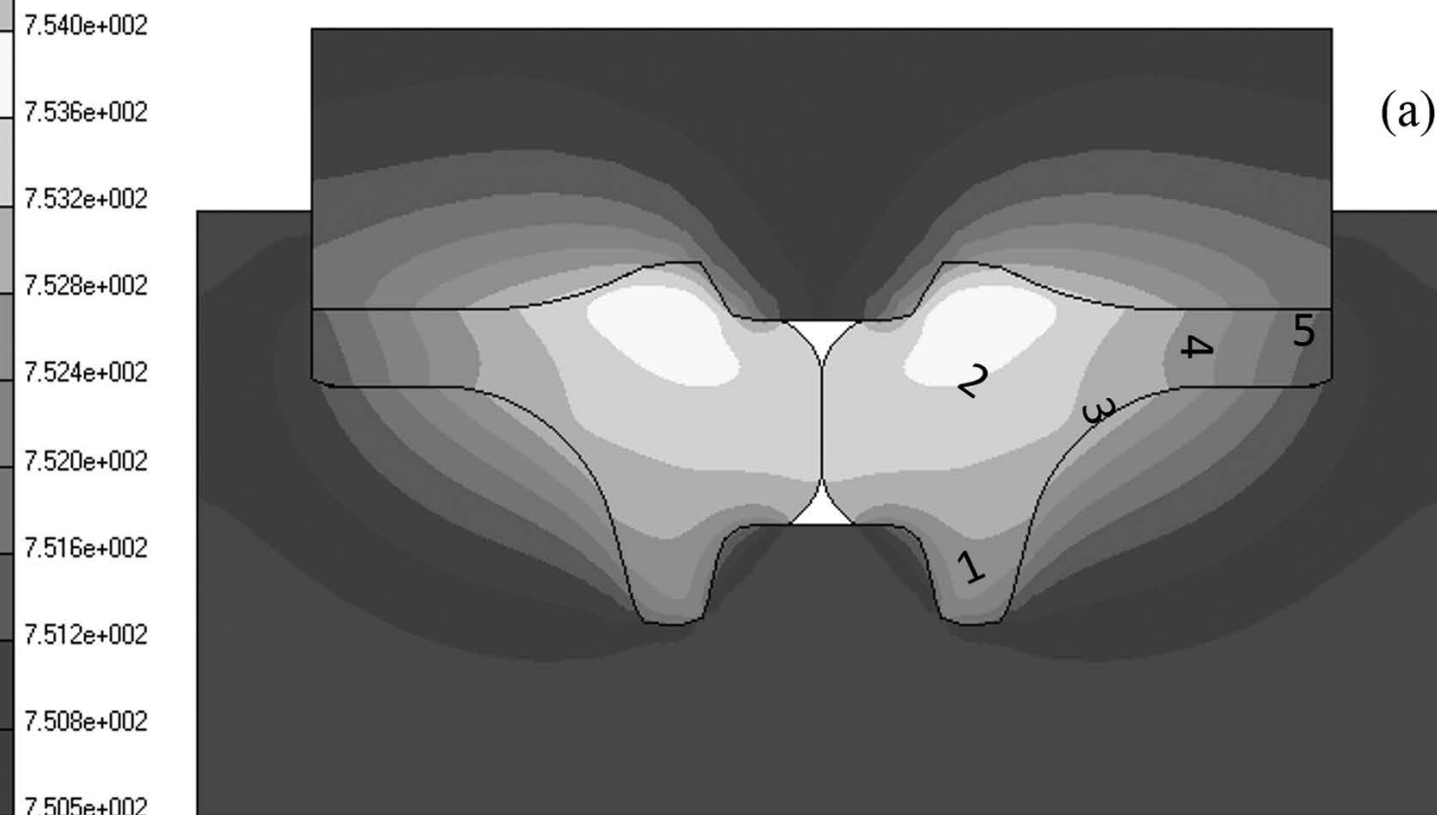

$7.505 e+002$

$7.501 \mathrm{e}+002$

Temoerature
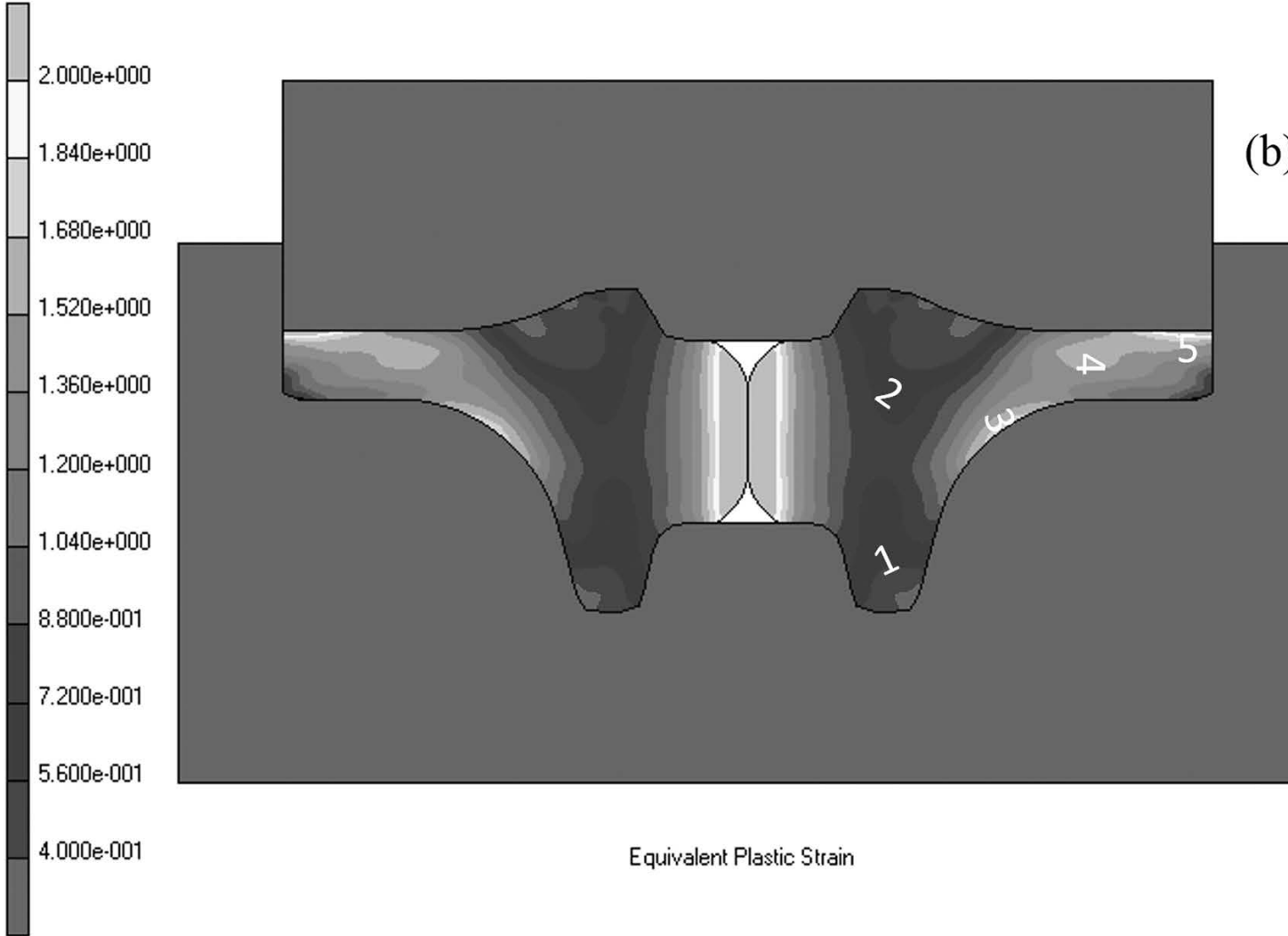

(b)

Figure 4. FEM simulation on the $\alpha / \beta$ forging process. Temperature (a) and equivalent plastic strain distribution (b) is shown with the gray counter on the left. Locations of different samples are marked with numbers in the picture 
Dense flow lines mean a large amount of nonmetallic inclusions inherited from the casting process and strong interactions between inclusion and prior grain boundaries. Thus, it is much easier to nucleate and grow in the prior- $\beta$ grain boundaries than between the prior- $\alpha$ plates.

Volume fractions of the primary $\alpha$ phase can be affected by multiple factors. Generally, low temperature and a high amount of deformation lead to more globularization of the $\alpha$ phase which results in a high volume fraction of the primary $\alpha$ phase. For $\mathrm{S} 1$, the temperature rather than the small deformation dominate the influence on the high volume fraction. In contrast, a large deformation rather than the temperature dominates the influence on the high volume fraction in S3. However, low temperature and a large deformation in S5 cannot explain why it has the lowest volume fraction of the primary $\alpha$ phase. In this case, sample position may play an important role. S5 is distant from the forging center where the metal flow arrives last when fulfilling the shape of the die. This means there is much less deformation time left for recrystallization, resulting in the lowest volume fraction in S5.

\subsection{The origin of texture inhomogeneity}

Texture inhomogeneity within the disk was observed with different texture sharpness and texture types. It can be easily found that the texture sharpness distribution has a great connection with strain distribution.
From Figure 4 (b) and Table 2, the equivalent plastic strain and the texture index of the $\beta$ phase show a similar variation trend with low values in S1 and S2 and rather high values in S3 and S5. It can be concluded that texture sharpness of the $\beta$ phase can be increased with deformation during forging. Thus, it is suggested that deformation may play the most important role in the texture inhomogeneity for the $\beta$ phase.

As for texture type of two phases, ODF analysis was added for more precise characterization. Figure 5 presents the ODF section at $\varphi 2=45^{\circ}$ for the $\beta$ phase and section at $\varphi 2=0^{\circ}$ for the $\alpha$ phase with the same range of intensity in each phase for comparison between samples. There are five types of major texture for the $\beta$ phase in the disk, i.e., $\{001\}$ $<110>,\{001\}<100>,\{111\}<110>, \gamma$-fiber, and $\alpha$-fiber, as indexed in the figure. They are all common rolling textures in bcc materials. However, two fiber textures found in S1 and S5, are not common in hot-working processed materials. In this case, the positions of these two samples may play an important role. They are both located at the rim of the disk, where interface friction occurs between the die and the material. This additional friction may account for the formation of observed fiber textures.

The volume fraction of each texture component for the $\beta$ phase in each sample was also calculated and is shown in Figure 5 (c). The rotated cube texture $\{001\}<110>$ is prominent in all the samples except S2. $\varphi 1 S 1$

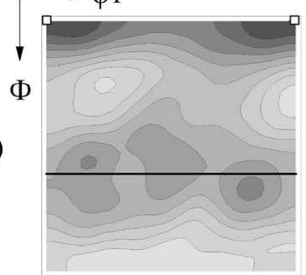

(b)

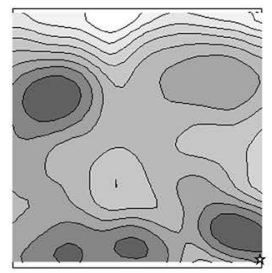

(c)

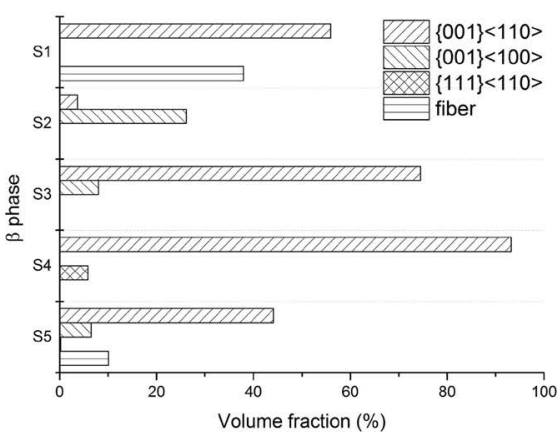

S3
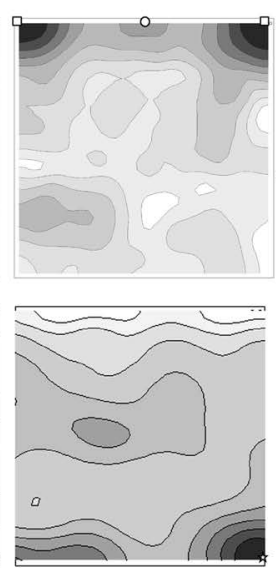

(d)

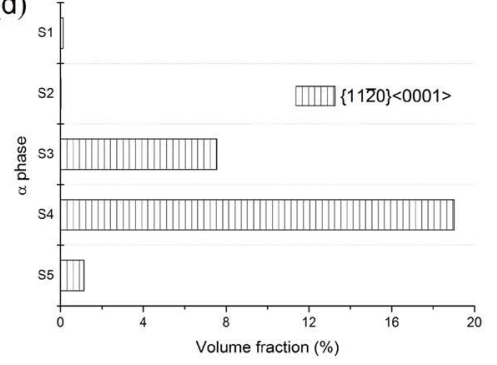

$\mathrm{S} 5$
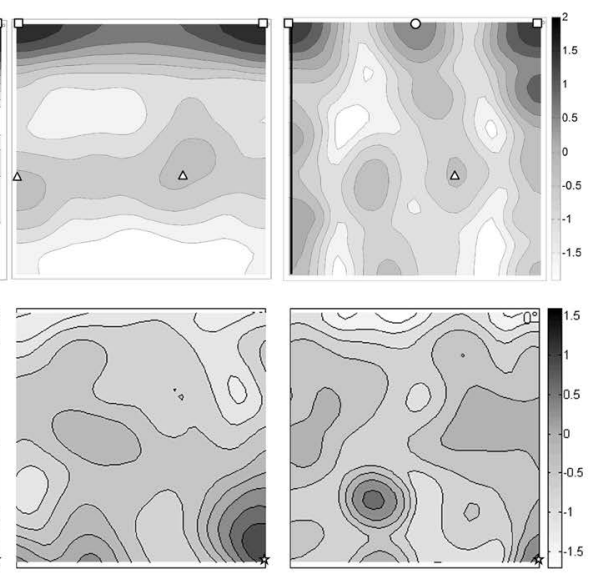

- $\{001\}<110>$

- $\{001\}<100>$

$\Delta\{111\}<110>$

* $\{1120\}<0001>$

- $\gamma$-fiber

$\alpha$-fiber

Figure 5. ODF section at $\varphi 2=45^{\circ}$ for the $\beta$ phase (a) and section at $\varphi 2=0^{\circ}$ for the $\alpha$ phase (b) represented by the color bar with the logarithmic scale in multiples of random distribution. The calculated volume fractions of different texture components for the $\beta$ phase (c) and for the $\alpha$ phase (d) 
The cube texture $\{001\}<100>$ is the prominent texture in $\mathrm{S} 2$ with a volume fraction of $\sim 25 \%$. It is well known that the cube texture is more prevalent in the former $\beta$ forging process. As discussed before, $\mathrm{S} 2$ bears high temperature and has a small deformation, resulting in a plate-like microstructure inherited from the former $\beta$ forging process. The major cube texture in S2 may also have been inherited from the former process; thus, it is also a result of high temperature and a small deformation. In addition, by comparing the volume fractions of major deformation texture components in $\{001\}<110>$, one can easily discover that the volume fraction in S5 is less than that in S3 and S4, despite that they all underwent a large extent of deformation. It is even less than the volume fraction in S1 which underwent much less deformation than S5. This can also be described by the position of S5, as it is the final part where metal flow arrives, resulting in there being much less time to deform. Therefore, as the two findings from texture analysis coincide with the findings from microstructure analysis, the origin of these two texture features is suggested to be the same as that of corresponding two microstructure features, making the formation mechanism more reliable.

As for the primary $\alpha$ phase, the transverse texture $\{11 \overline{2} 0\}$ $<0001>$ characterized from pole figures indicates the primary $\alpha$ phase also contributes to the deformation texture with the $\beta$ phase. Thus, to investigate the relationship of transverse texture and deformation, only the ODF section at $\varphi 2=0^{\circ}$ for the $\alpha$ phase with marked transverse texture was used for distinct perception. The transverse texture is prominent in $\mathrm{S} 3$, $\mathrm{S} 4$, and S5, and disappeared in S2. The calculated volume fraction of the transverse texture is shown in Figure 5 (d). The low values in S1 and S2 and the high values in S3, S4, and S5 also show a strong connection with the strain distribution. This means that large deformations are more likely to lead to the development of the transverse texture. In addition, texture sharpness of the primary $\alpha$ phase is much lower than the $\beta$ phase, presumably because of the constraint imposed by the surrounding $\beta$ matrix.

\section{Conclusions}

The study analyzed the effect of final $\alpha / \beta$ forging on the microstructure and texture variations in a Ti-1023 forged disk. Microstructure variations within the disk show a strong connection with the forging condition. The formation of a plate-like primary $\alpha$ phase can be attributed to the high temperature and small deformation. The necklace-like structure of the primary $\alpha$ phase forms in the regions with the highest density of flow lines. Volume fractions of the primary $\alpha$ phase can be affected by both temperature and deformation. Texture variations are dominated by the deformation of the $\beta$ phase. Texture sharpness of the $\beta$ phase shows a similar variation trend to strain distributions. Common rolling textures for the $\beta$ phase and the transverse texture for the $\alpha$ phase were observed with different volume fractions in different regions of the disk.
Two $\beta$ fiber texture components, $\gamma$-fiber, and $\alpha$-fiber, observed in regions near the rim of the disk can be attributed to the interface friction between the die and the material. The weak cube texture observed in the central part of the disk is a result of high deformation temperature. The transverse texture for the primary $\alpha$ phase is more favored by large deformation.

\section{Acknowledgements}

This work was supported by the National Natural Science Foundation of China (Nos. 51771049 and 51790484), State Key Lab of Advanced Metals and Materials (No. 2016Z06) and the Fundamental Research Funds for the Central Universities (Grant No. N150204002 and N160204002).

\section{References}

1. Boyer R, Collings EW, Welsch G, eds. Materials Properties Handbook: Titanium Alloys. Materials Park: ASM International;1994.

2. Peters M, Kumpfert J, Ward CH, Leyens C. Titanium Alloys for Aerospace Applications. Advanced Engineering Materials. 2003;5(6):419-427.

3. Matthew JD. Titanium: A Technical Guide. Materials Park: ASM International; 2000

4. Singh S, Singh R. Precision investment casting: A state of art review and future trends. Proceedings of the Institution of Mechanical Engineers, Part B: Journal of Engineering Manufacture. 2016;230(12):2143-2164.

5. Fang ZZ, Paramore JD, Sun P, Ravi Chandran KS, Zhang Y, $\mathrm{Xia} Y$, et al. Powder metallurgy of titanium - past, present, and future. International Materials Reviews. 2018;63(7):407-459.

6. Li N, Kong HP, Du BR, Liu CK. Analysis of Microstructure and Defects of FGH96 Superalloy Manufactured by Laser Rapid Prototyping. Failure Analysis and Prevention. 2016;11(2):124-128.

7. Altan T, Ngaile G, Shen G, eds. Cold and Hot Forging: Fundamentals and Applications. Volume 1. Materials Park: ASM International; 2004.

8. Lutjering G, Williams JC. Titanium. Engineering Materials and Processes. Berlin: Springer; 2007.

9. Duerig TW, Williams JC. Overview: Microstructure and properties of Beta-Titanium. In: Boyer RR, Rosenberg HW, eds. Beta-titanium alloys in the 1980 's. Warrendale: TMS AIME; 1984. p. 19-69.

10. Kolli RP, Devaraj A. A Review of Metastable Beta Titanium Alloys. Metals. 2018;8(7):506

11. Raghunathan SL, Dashwood RJ, Jackson M, Vogel SC, Dye D. The evolution of microtexture and macrotexture during subtransus forging of Ti-10V-2Fe-3Al. Materials Science and Engineering: A. 2008;488(1-2):8-15.

12. Gey N, Bocher P, Uta EL, Humbert GM. Texture and microtexture variations in a near-a titanium forged disk of bimodal microstructure. Acta Materialia. 2012;60(6-7):26472655 . 
13. Chateigner D. Combined Analysis. London, Hoboken: ISTEWiley; 2010.

14. Lutterotti L. Maud: a Rietveld analysis program designed for the internet and experiment integration. Acta Crystallographica Section A. 2000;56(Suppl 1):s54.

15. Burgers WG. On the process of transition of the cubicbody-centered modification into the hexagonal-close-packed modification of zirconium. Physica. 1934;1(7-12):561-586.
16. Bunge HJ. Texture Analysis in Materials Science: Mathematical Methods. London: Butterworths; 1982.

17. Lin HZ, Guo L, Lian JM, Huang X. The FEM simulation and analysis for the Ti-1023 hub forgings' process. Journal of Aeronautical Materials. 2003;23(Suppl):95-99.

18. Weiss I, Froes FH, Eylon D, Welsch GE. Modification of alpha morphology in Ti-6Al-4V by thermomechanical processing. Metallurgical and Materials Transactions A. 1986;17(11):1935-1947. 